$\mathrm{V}$ (NF1), first described about 100 years ago, is one of the more frequent autosomal dominant mutations of humans 1,2 . Although very little is known about the basic biology of the disease, genetic analysis has proceeded rapidly over the past three years as a result of linkage analysis and physical mapping techniques ${ }^{3}$. The purpose of this brief review is to describe these advances and to identify future challenges. Much of this information was recently discussed at a meeting of the International Consortium for NF Gene Cloning, sponsored by the National Neurofibromatosis Foundation in February 1989, and we owe much gratitude to the Foundation and the participants in that consortium for permission to present this synthesis.

\section{Clinical features of NF1}

The diagnostic criteria for NF1, as recently enumerated by an NIH consensus conference ${ }^{4}$, are listed in Table 1. A diagnosis is usually established by the finding of multiple café-au-lait spots and cutaneous neurofibromas (Fig. 1). Establishing a diagnosis in children may be difficult if multiple café-au-lait spots are not present, as the neurofibromas often do not make their appearance until adolescence. Besides the diagnostic criteria listed in Table 1 , a variety of other complications can also occur, ranging from milder difficulties such as itching and constipation to more serious problems such as seizures, learning disability, or even occasionally frank mental retardation, major orthopedic abnormalities, and the development of malignancy, particularly neurofibrosarcoma or optic glioma.

\section{Genetics}

The landmark study of Crowe, Schull and Neel ${ }^{1}$ in the 1950s demonstrated that NF1 is inherited in an autosomal dominant fashion. Penetrance is extremely high if careful attention is paid to the diagnostic criteria listed in Table 1, and there are few examples of genuine nonexpressing individuals. However, variable expressivity is the rule in this disorder, with a dramatic variation in severity between affected individuals. This variation is in general as great within a family as between families, indicating that it is not simply a result of genetic heterogeneity. The incidence of the disorder is about 1 in 3000 in all races that have been carefully examined ${ }^{2}$. A remarkable feature is the high spontaneous mutation rate, with $40-50 \%$ of all cases representing spontaneous mutations. This leads to a calculated mutation rate of $10^{-4}$ per allele per generation, among the highest such rates reported for a locus in humans. This may have important implications for the structure of the NF1 locus.

In the past there has been some clinical and genetic confusion between NF1 and a related disorder, bilateral acoustic neurofibromatosis (NF2). In NF2 a few café-au-lait spots may be present, and occasionally a small number of peripheral neurofibromas will also appear. The hallmark of NF2, however, is the presence of bilateral acoustic neuromas of the eighth cranial nerve $^{3}$, which appear in more than $90 \%$ of individuals with this gene by age 30 . Acoustic neuromas are
Progress towards identifying the neurofibromatosis (NF1) gene

\section{FRANCIS S. COLIINS, PETER O'CONNELL, BRUCE A.J. PONDER AND BERND R. SEIZINGER}

Von Recklingbausen neurofibromatosis (NF1) is a common autosomal dominant disorder of bumans. Linkage analysis bas recently mapped the NF1 gene to the proximal long arm of cbromosome 17. The identification of two NF1 patients witb balanced translocations bas now allowed the location of the gene to be narrowed to a few bundred kilobases of chromosome band 17q11.2, using a combination of somatic cell bybrid technology, linking clones and pulsed field gel electropboresis.

extremely rare in NF1. Although occasional families have been reported in which individuals appear to have features of both disorders, the successful mapping of NF1 to chromosome 17 (Refs 5,6 ) and NF2 to chromosome 22 (Ref. 7) provides conclusive proof that these are different disorders

\section{Linkage analysis of NF1}

The relatively high frequency of the disorder, coupled with its high penetrance and associated high diagnostic accuracy, made NF1 an attractive target for linkage analysis as soon as sufficient panels of polymorphic markers emerged. This effort, initiated by several groups who worked together to establish an exclusion map, resulted in the identification of definitive linkage to the pericentromeric region of chromosome 17 in the late spring of 19875,6. At the time of writing, no families with classic NF1 have been identified that do not show linkage to this region; therefore, it is likely that this disease represents mutation at a single locus.

Over the past year and a half, a large number of additional polymorphic markers have been generated

\section{TABIE 1. Diagnostic criteria for NF1}

Two or more of the following:

(1) $\geq 6$ Café-au-lait spots $\geq 1.5 \mathrm{~cm}$ in postpubertal individual $\geq 0.5 \mathrm{~cm}$ in prepubertal individual

(2) $\geq 2$ Neurofibromas of any type, or $\geq 1$ plexiform neurofibroma (a deeply placed, large and complex neurofibroma)

(3) Freckling of the armpits or groin

(4) Optic glioma (tumor of the optic nerve)

(5) $\geq 2$ Lisch nodules (benign hamartomas of the iris)

(6) A distinctive bony lesion - dysplasia of the sphenoid bone - dysplasia or thinning of long bone cortex

(7) First degree relative with NF1 
from chromosome 17 , and these probes have been tested by the various groups involved in linkage analysis, in a productive collaborative effort sponsored by the National Neurofibromatosis Foundation. In a series of published reports, first in Genomics and then in the American Journal of Human Genetics, an intense genetic analysis of this disorder was carried out. The genetic mapping effort culminated in the multipoint linkage map shown in Fig. 2, which was constructed by David Goldgar and colleagues from the collaborative group's efforts'. This multipoint map represents the outcome of the study of 142 families with over 700 affected individuals, and a total of 13838 genotypings. It is apparent from Fig. 2 that probes on either side of NF1 are now available within less than $5 \mathrm{cM}$ of the gene, so that application of DNA technology to prenatal or

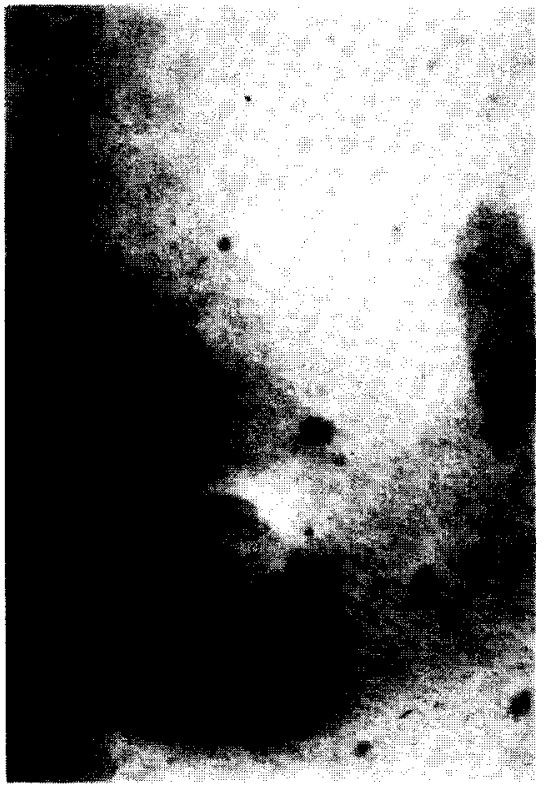

$F I G \mathbf{1}$

Typical appearance of the skin in an individual with NF1. A café-au-lait spot, a large neurofibroma, and several small neurofibromas are apparent

NF1 and balanced translocations

As expected for an autosomal dominant disorder, cytogenetic analysis of patients with NF1 has in general been quite unrevealing. However, two unique patients have been identified who have NF1 in association with apparently balanced translocations ${ }^{10.11}$ and these have played a critical role in fine mapping of the gene.

This is perhaps an important lesson for reverse genetics of other diseases: the identification of such cytogenetic abnormalities, even if they represent only a rare mechanism of causation for a particular disease, can greatly accelerate the identification of the responsible region. Such cytogenetic abnormalities have, for example, aided the identification of the Duchenne muscular dystrophy ${ }^{12}$, chronic granulomatous disease $^{13}$, retinoblastoma ${ }^{14}$ and sex determination loci' ${ }^{15}$, and the presymptomatic diagnosis of this disease is now possible.

This genetic analysis thus places NF1 on the proximal long arm of chromosome 17. Initial surveys of this region revealed at least three candidate genes: two of these, the nerve growth factor receptor and the erbAl proto-oncogene, were quickly shown to map distal to NF1 by the identification of recombinant individuals ${ }^{6,9}$ : a third candidate gene, the erbB2 proto-oncogene (also sometimes called NEU, HER2 or $N G L$ ), has not yet yielded a polymorphism and so could not be mapped genetically. Subsequent physical mapping experiments, however. have now also excluded this locus (see below).

\section{$F I G \mathbf{2}$}

Multipoint linkage map of the NF1 region of chromosome 17 (from Ref. 8). The alpha satellite marker D17Z1 indicates the position of the centromere. The order of markers shown is favored by at least 100:1 odds over alternatives that involve interchanging a pair of adjacent markers. The genetic distances (in centimorgans) are sex-averaged; in this region of chromosome 17 , as in much of the human genome, genetic distances are about two to three times greater in female meiosis than in male meiosis. absence of such abnormalities has hindered the rapid identification of the cystic fibrosis ${ }^{16}$ and Huntington disease $^{17}$ loci.

A diagram of the cytogenetic abnormalities identified in the two unique NF1 patients is shown in Fig. 3. In one situation ${ }^{10}$ an apparently balanced translocation has occurred between 1 p34.3 and 17q11.2. In the other situation ${ }^{11}$. the translocation involves $17 q 11.2$ and $22 q 11.2$. The fact that one breakpoint in both instances involves $17 q 11.2$, precisely where the NF1 gene has been mapped by linkage analysis, is strong circumstantial evidence that these translocations are in fact causative rather than coincidental.

\section{Mapping with somatic cell hybrids}

The convenient localization of the selectable marker thymidine kinase (TK) on distal $17 \mathrm{q}$ has greatly facilitated the creation of a robust panel of somatic cell hybrids involving various segments of chromosome 17. Such a panel is shown in Fig. 4, although this represents only a subset of the hybrids created by Ledbetter ${ }^{11}$ and by Leach and Fournier ${ }^{1 x}$. Particularly noteworthy in Fig. 4 are the hybrids denoted DCR-1 and NF13, which are respectively derived from the $t(1 ; 17)$ and $t(17 ; 22)$ patients. Thus, the endpoint of these two hybrids, which occur at the interface between the regions denoted as region 2 and region 3 on the diagram, should indicate the location of the NF1 gene. In fact, the panel shown in the figure can be used to divide the long arm of chromosome 17 into five regions. Mapping the genetic markers used for the multipoint analysis shown in Fig. 2 against this somatic cell hybrid panel gives consistent results, as shown in Fig. 4. This adds additional confidence to the genetic map, indicates that the hybrid mapping panel does not apparently contain major chromosome rearrangements. 
and further supports the argument that the translocations contained in NF13 and DCR1 are causative of the disease, since they map to precisely the right interval. This combination of approaches thus provides very useful complementary information.

\section{Physical mapping}

The identification of a translocation breakpoint is directly approachable using pulsed field gel electrophoresis (PFGE). What one requires is a probe that resides on a restriction fragment altered by the translocation. Since the rare-cutting restriction enzymes used in this sort of analysis often generate fragments of several hundred kilobases or more, the probe used does not have to be precisely at the translocation breakpoint in order to identify its presence. Preliminary efforts with the most closely linked genetic markers (HHH202, TH17.19 and EW206) did not, however, reveal any abnormalities in PFGE analysis of the two translocation patient DNAs ${ }^{19}$. This analysis did, however, indicate that $\mathrm{HHH} 202$ and $\mathrm{TH} 17.19$ lie only about a hundred kilobases apart (not surprising since TH17.19 was cloned from a PFGE fragment detected by HHH202; Ref. 18), and also somewhat surprisingly that EW206 and EW207 can be no more than $700 \mathrm{~kb}$ apart ${ }^{19}$. This latter finding was unexpected given the approximate $4 \mathrm{cM}$ genetic distance between these probes, which would have predicted a physical separation of about 4 million base pairs. This may indicate the possibility of a hot spot for recombination between these two probes, although the possibility of data errors or incorrect paternity also needs to be excluded.

To identify the translocation breakpoints on physical maps, several groups have now identified more DNA markers in this region of chromosome 17 , and two groups have now identified markers capable of visualizing the translocation breakpoints by PFGE analysis. Wallace et al. prepared a series of chromosome-17-specific NotI linking clones ${ }^{20}$, and used the somatic cell hybrid mapping panel in Fig. 4 to identify clones in regions 2 and 3 . One of their region 2 clones, denoted 17L1, detects PFGE abnormalities in the $t(1 ; 17)$ patient and in her affected offspring ${ }^{21}$. The presence of abnormal fragments with a variety of different enzymes and in several different tissues pro-

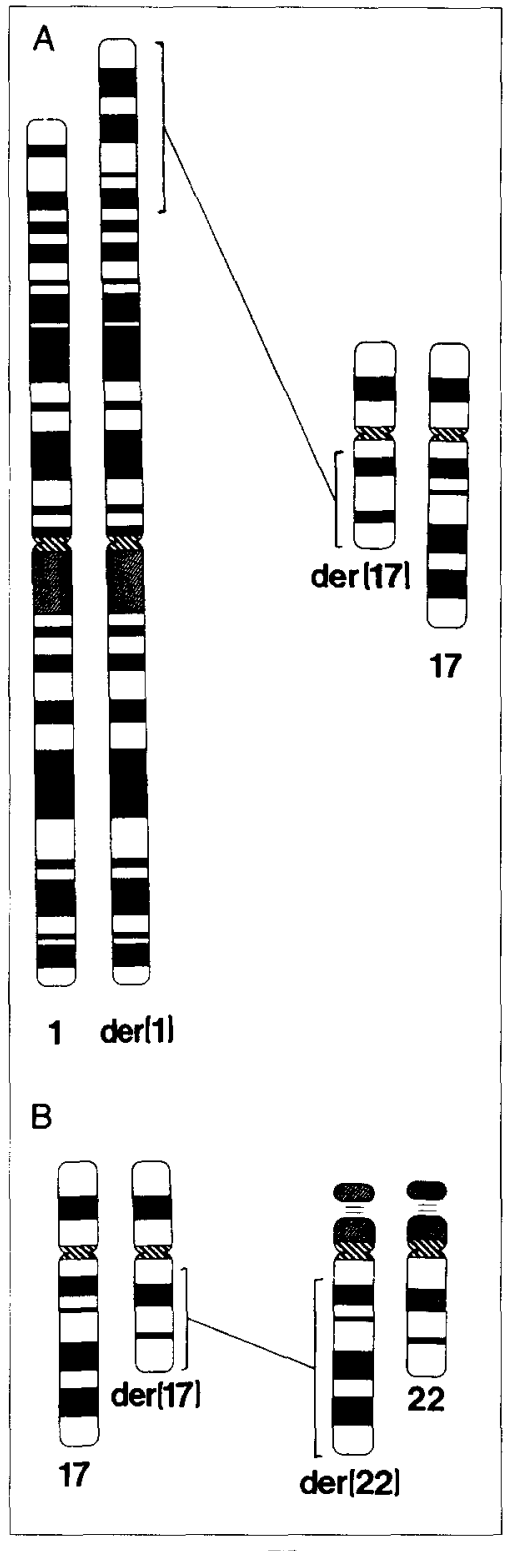

\section{$F I G \mathbf{3}$} with NF1. (A) $t(1 ; 17)(\mathrm{p} 34.3 ; \mathrm{q} 11.2)$. (B) chromosomes are shown Note that in both patients one breakpoint involves 17 q11.2, the precise location of NF1 by linkage analysis vides conclusive evidence that this translocation breakpoint maps near $17 \mathrm{~L} 1$, and was used to construct the physical map in Fig. 5. The $\mathrm{t}(17 ; 22)$ breakpoint is slightly further away, as shown in the figure. Both these breakpoints are telomeric to $17 \mathrm{~L} 1$, since this probe is absent in the NF13 and DCR-1 hybrids.

O'Connell et al. ${ }^{18}$ mapped a large number of cosmids to regions 2 and 3 . One of these, denoted c11-1F10, detects abnormal PFGE fragments with both translocation patients ${ }^{22}$, and maps to region 3. Its approximate localization on the physical map is also shown in Fig. 5. Thus these two cloned probes, which reside on the same $600 \mathrm{~kb}$ NruI fragment, bracket the two translocations

\section{Future work}

The combination of genetic and physical mapping techniques has thus dramatically narrowed the potential localization of the NF1 gene to a region of only a few hundred kilobases in band q11.2 of chromosome 17 . The fact that the two translocation breakpoints are separated by a minimum of $60 \mathrm{~kb}$, based on the physical map in Fig. 5 , indicates that the NF1 gene is of at least moderate size. This sort of analysis does not, of course, place an upper limit on the size. In light of the very high spontaneous mutation rate, it is not impossible that the neurofibromatosis gene might be a 'megagene' analogous to the Duchenne muscular dystrophy gene, since that locus displays a similarly high mutation rate, presumably because its $2000 \mathrm{~kb}$ transcription unit provides a broad target for mutation. With this in mind, it is perhaps somewhat surprising that thus far no PFGE abnormalities have been identified in sporadic NF1 patients with the 17L1 or 1 F10 probes 21.22 .

The presence of a strong CPG island between the two translocation breakpoints (Fig. 5) is an interesting feature, since such GC-rich hypomethylated islands often mark the $5^{1}$ end of expressed genes ${ }^{23}$. It is somewhat difficult, however, to understand how both translocations could alter the expression of such a gene, since one of the translocation breakpoints would have to lie in the 5 ' flanking region at a considerable distance. The recent identification of a transcribed gene within a large intron of the factor VIII gene (J. Gitschier, pers. commun.) points out, however, 


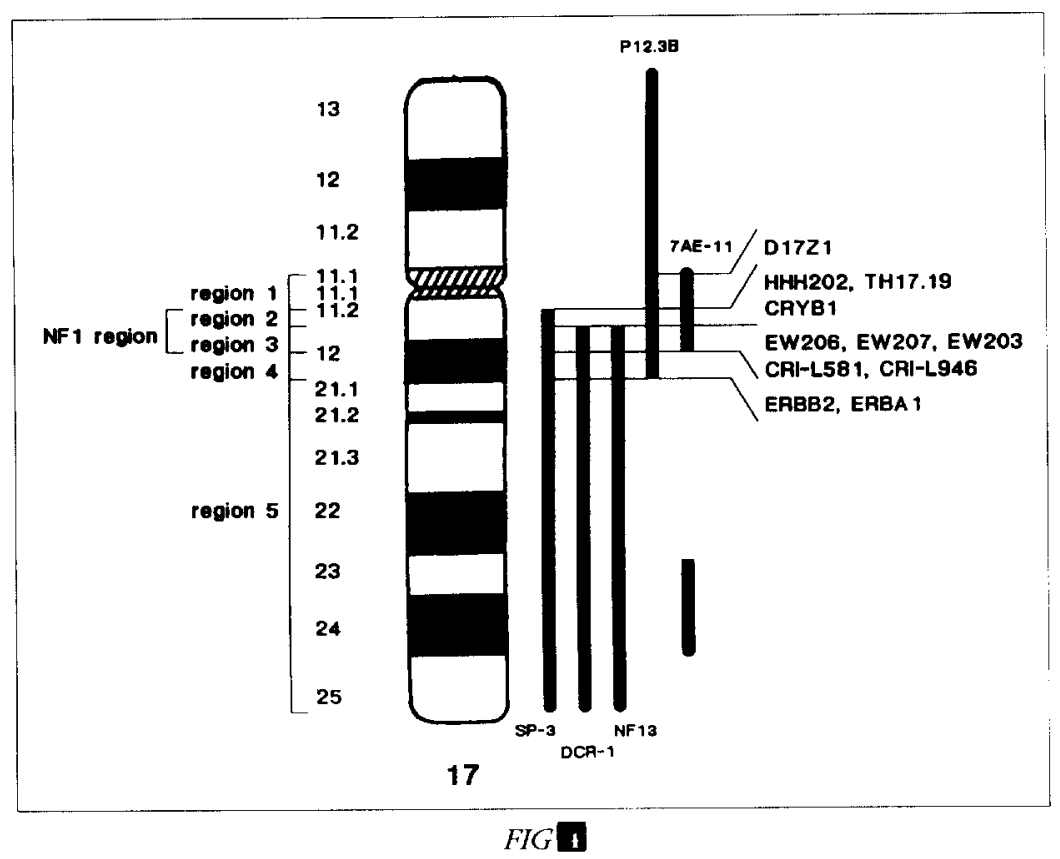

Mapping of DNA markers near NF1 with a panel of somatic cell hybrids ${ }^{11.18 .19}$. The portion of human chromosome 17 present in each hybrid is indicated by a black bar. SP-3 and P12.3B were derived from non-NF1 cell lines carrying chromosome 17 translocations. DCR-1 is a hybrid carrying the der $(1)$ chromosome from the $t(1 ; 17)$ NF1 patient whose chromosome rearrangement is shown in Fig. 3; NF13 carries the der(22) chromosome from the $t(17 ; 22) \mathrm{NF} 1$ patient. $7 \mathrm{AE}-11$ is a hybrid created using microcell-mediated transfer ${ }^{18}$, and has also been an important cloning resource. This set of hybrids can be used to subdivide the area around NF1 into five regions. A

probe is then placed in an interval by Southern blot analysis with each member of

the hybrid panel. Note that the derived order of markers agrees with the genetic map (Fig. 2). The NF1 gene is presumably located at the interface between regions 2 and 3, at the breakpoints of DCR-1 and NF13. northern blots, and cDNA library screening.

One of the potential difficulties in identifying the NF1 transcript is an uncertainty about its normal function, or even its usual tissue of expression. The peripheral neurofibromas which are such a characteristic feature of the disease are made up primarily of Schwann cells and fibroblasts, but analysis of clonality has indicated that these tumors are not clonal ${ }^{24}$, implying that they may be reacting to some local stimulatory influence. While most of the features of the disease occur in the neural crest, transcript identification may turn out to be more difficult than was the case for Duchenne muscular dystrophy or retinoblastoma. It is not clear, for example, whether the normal transcript or the abnormal transcript, or both, will be expressed in neural crest-derived tissues.

There are some features of NF1 that suggest that the gene is a tumor suppressor gene, analogous to the retinoblastoma or Wilms' tumor genes. In this model, the disease gene is actually recessive at the cellular level, although the inherited predisposition is dominant. This would be consistent with the fact that most cells of an affected individ-

that the situation may be complex. Efforts are currently underway using chromosome jumping, chromosome walking, and selective cloning from specific PFGE fragments to identify additional DNA markers within the region of the translocations. It will be important to determine whether these are simple translocations, or whether loss of material has occurred, as occasionally has been found in such situations. The focus now shifts to an attempt to identify transcripts from within this region. using a combination of 'zoo blots' (Southern blots containing DNA from different species, to assess sequence conservation), ual are apparently normal, with the enormous variable expressivity of the disease potentially arising from somatic genetic events required to produce actual cellular abnormalities. In such a model, the nonclonal benign neurofibromas would presumably be responding to a clonal abnormality of a nearby cell or group of cells, or even to the nerve axon.

A standard method of inferring the presence of such tumor suppressor genes has been the observation of allele losses at the affected locus in tumors from such individuals 25 . While benign neurofibromas are useless for such analyses in light of their nonclonality,

\section{$F I G \mathbf{5}$}

Physical map of the region around the two NF1 translocation breakpoints. 17L1A is a subclone of the Notl linking clone 17Ll (Ref. 21) and c11-1F10 is a cosmid 22 ; both detect abnormal pulsed field gel fragments in the NF1 translocation patient DNAs. Both probes are on the same $600 \mathrm{~kb}$ Nril fragment, and they flank the translocations, the location of which is indicated by crosshatching. Sizes are in kilobases. Key: $M$, MluI; N, Notl; L, NruI; B, BssHII; S, SacII.

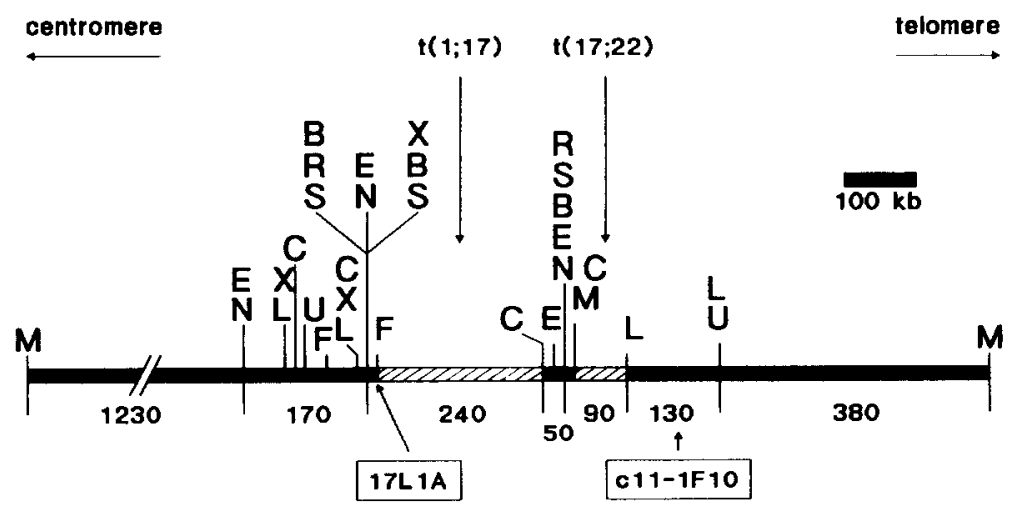

Chromosome 17 
several groups have analysed malignant tumors arising in NF1 patients, particularly neurofibrosarcomas, and have identified a high frequency of allele loss on chromosome 17. The fact that there is a known tumor suppressor gene (most likely the P53 gene26) on 17p, however, makes this analysis quite complicated, as the reduction to homozygosity has in general involved the entire chromosome, and has occasionally even involved $17 \mathrm{p}$ but not $17 \mathrm{q}$ (B. Seizinger and T. Glover, unpublished). Thus, at present, there is no strong biologic evidence to support the tumor suppressor gene mechanism for NF1.

\section{Conclusion}

Analysis of NF1 has proceeded rapidly in the past two years, with localization of the gene now narrowed to only $0.01 \%$ of the genome. The availability of translocations has played a critical role in this process, and should now greatly assist the identification of a candidate transcript, which may well be possible in the next one to two years. The results are likely to shed important light on the biology of the neural crest, the mechanism of tumor formation, and the nature of this puzzling and dramatic disorder.

\section{Acknowledgements}

The continuing support of the National Neurofibromatosis Foundation and its International Consortium for NF1 Research is greatly appreciated. Support from the European Organization LINK is also acknowledged with gratitude. The authors thank Jane Fountain for providing some of the figures, Peggy Wallace for helpful discussions and Bernice Sandri for preparing the manuscript. F.S.C. acknowledges support from the NIH in the form of grants NS23410 and NS23427; F.S.C. is an Associate Investigator of the Howard Hughes Medical Institute.

\section{References}

1 Crowe, F.W., Schull, W.T. and Neel, V.F. (1956) $A$ Clinical, Patbological, and Genetic Study of Multiple Neurofibromatosis, Charles C. Thomas, Springfield

2 Riccardi, V.M. and Eichner, J.E. (1986) Neurofibromatosis: Phenotype, Natural History, and
Pathogenesis, Johns Hopkins University Press

3 Collins F.S., Ponder, B.A.J., Seizinger, B.R. and Epstein, C.J. (1989) Am. J. Hum. Genet. 44, 1-5

4 Stumpf, D.A. et al. (1987) Neurofibromatosis ( $N I H$ Consensus Development Conference Statement) Vol. 6 , no. 12

5 Barker, D. et al. (1987) Science 236, 1100-1102

6 Seizinger, B.R. et al. (1987) Cell 49, 589-594

7 Rouleau, G.A. et al. (1987) Nature 329, 246-248

8 Goldgar, D.E., Green, P., Parry, D.M. and Mulvihill, J.J. (1989) Am.J. Hum. Genet. 44, 6-12

9 Seizinger, B.R. et al (1989) Am. J. Hum. Genet. 44, 30-32

10 Schmidt, M.A., Michels, W. and Dewald, W. (1987) Am. J. Med. Genet. 28, 771-777

11 Ledbetter, D.H. et al. (1989) Am.J. Hum. Genet. 44, $20-24$

12 Monaco, A.P. et al. (1986) Nature 323, 646-650

13 Royer-Pokora, B. et al. (1986) Nature 322, 32-38

14 Friend, S.H. et al. (1986) Nature 323, 643-646

15 Page, D.C. et al. (1987) Cell 51, 1091-1104

16 Iannuzzi, M.C. et al. (1989) Am. J. Hum. Genet. 44, 695-703

17 Wasmuth, J.J. (1988) Nature 332, 734-736

18 O'Connell, P. (1989) Am.J. Hum. Genet. 44, 51-57

19 Fountain, J. (1989) Am. J. Hum. Genet. 44, 58-67

20 Wallace, M.R., Fountain, J.W., Brereton, A.M. and Collins, F.S. (1989) Nucleic Acids Res. 17, 1665-1677

21 Fountain, J. et al. (1989) Science 244, 1085-1087

22 O'Connell, P. et al. (1989) Science 244, 1087-1089

23 Bird. A.P. (1986) Nature 321, 209-213

24 Fialkow, P.J., Sagebriel, R.W., Gartler, S.M. and Rimoin, D.L. (1971) New' Engl. J. Med. 284, 298-300

25 Vogelstein, B. et al. (1989) Science 244, 207-210

26 Baker, S.J. et al. (1989) Science 244, 217-220

F.S. Colluns is in the Howard Hughes Medical Institute, DEPARTMENTS OF HUMAN GENETICS AND INTERNAL MEDICINE, UNIVERSITY OF MICHIGAN, ANN ARBOR, MI 48109, USA; P. O'CONNELL IS IN THE HowaRd Hughes MEDICAL INSTTUTte, AND DEPARTMENT OF HUMAN GENETICS, UNIVERSTYY OF UTAH, SALT LAKE CITY, UT 84132, USA; B.AJ. PONDER IS IN THE

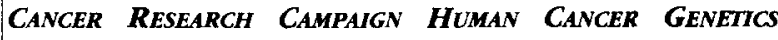
RESEARCH GROUP, UNIVERSITY OF CAMBRIDGE, UK; BR. SEIZINGER IS IN THE MasSACHUSETTS GENERAL HOSPITAL, HARVARD MEDICAL SCHOOL, BOSTON, MA 02114, USA.

\section{Trends in Genetics Centre-page diagrams: The polymerase chain reaction}

\section{(Scientific advisen: Thomas J. White, Norman Arnbeim and Henry A. Erlicb)}

Extra copies of the colour poster-style illustration of the polymerase chain reaction published last month in $T G$ are available. Centrepage diagrams are sold in multiples of six - either six of one, or a selection of six from $7 G$ and its sister journals Imminology Today,

Trends in Biotechnology, Trends in Ecology and Evolution, Trends in Pharmacological Sciences and Trends in Neurasciences

- IIG The polymerase chain reaction (Ref. 91)

- TREE DNA fingerprinting and RFLP analysis (Ref E3)

- $\Pi$ : Computer models of human inmunoglobulins and TCRs (Ref 15 , two diagrams, counts as two choices)

- IT The pleiotropic effects of B-cell factors (Ref. 17)

- IT: The mocaic of autoimmunity (Ref. 19)

- TIPS Phamacology of lipoprotein metabolism (Ref. 67)

- IINS The development of Drosopbila melangaster (Ref. 56)
- TBTECH. Second generation monoclonal antibodies (Ref. TT3)

- IT: Sequence patterns in domains of the immunoglobulin superfamily (Ref. 16)

- IT. Murine hematopoietic cell surface antigen expression (Ref. 18) - TIPS: Transmembrane signalling (Ref 66)

- TIPS. Types of opioid receptor (Ref. 68)

- IINS Structure-function relationships in vettebrate retina (Ref. 58)

Prices (for six copies): $\$ 8.00(+15 \%$ VAT in UK) $\$ 15.00$

Prices include postage and packing. Large orders (100 copies or mofe in total) are available at a $15 \%$ discount

Send your order, with payment, to:

Centre-Page Diagrams, Elsevier Science Publishers Ltd, Crown House, Linton Road, Barking, Essex IG11 8JU, UK. 